\title{
Supporting Information: Bioaccumulation dynamics of arsenate at the base of aquatic food webs
}

Adeline R. Lopez ${ }^{1}$, Dean R. Hesterberg ${ }^{2}$, David H. Funk ${ }^{3}$, David B. Buchwalter ${ }^{4}$ *

1 Supporting information is 4 pages in length and includes one figure, one table, and description of

2 methods for aqueous uptake in Neocloeon triangulifer. 


\section{Supplemental Information}

A

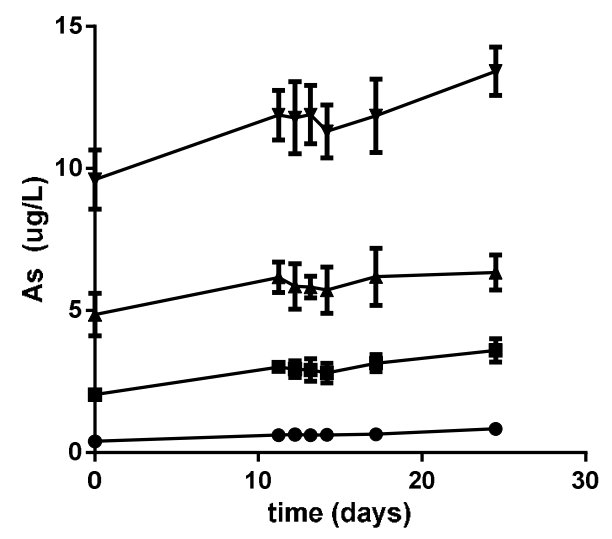

B

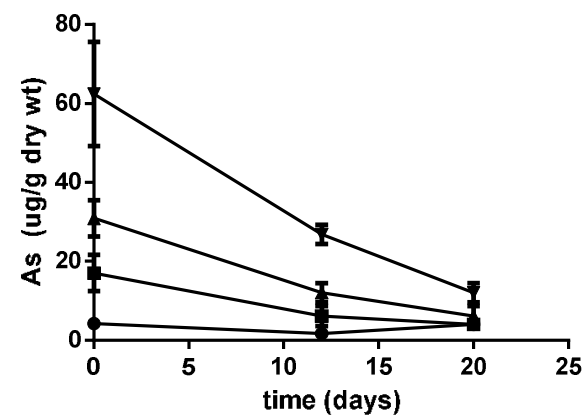

Supplemental Figure 1. Aqueous (A) and dietary (B) exposure conditions for $N$. triangulifer larvae reared for a full lifecycle. Values plotted are mean \pm SEM; $n=3$ for each treatment at each time point. Symbols represent initial nominal dissolved As concentrations of 1, 5, 10, and $20 \mu \mathrm{g} \mathrm{L}^{-1}$ arsenate, respectively. 


\section{Supplemental Table 1. Species composition of periphyton plates evaluated}

June 2009 - December 2009

\begin{tabular}{|c|c|c|c|}
\hline & \multicolumn{3}{|c|}{ Month } \\
\hline & June-July & October & December \\
\hline \multirow[t]{15}{*}{ Diatoms $^{1}$} & Melosira varians & Melrosira varians & Melosira varians \\
\hline & Diatoma vulgaris & Cymbella sp. & Gomphonema sp. \\
\hline & Synedra sp. & Synedra sp. & Nitzschia sp. \\
\hline & Nitzschia sp. & Nitzchia sp. & Synedra sp. \\
\hline & Cymbella sp. & Navicula sp. & Fragilaria $s p$. \\
\hline & Gomphonema sp. & Achnanthidium sp. & Cymbella sp. \\
\hline & Fragilaria $s p$. & Planothidium $s p$. & Navicula sp. \\
\hline & Navicula sp. & Frustulia sp. & Diatoma sp. \\
\hline & Achnanthidium sp. & Cocconeis sp. & Achnanthidium sp. \\
\hline & Diadesmis sp. & Fragilaria $s p$. & Asterionella sp. \\
\hline & Diatoma sp. & Diatoma sp. & Meridion $s p$. \\
\hline & Brachysira $s p$. & Gomphonema sp. & Cyclotella sp. \\
\hline & Rhoicosphenia sp. & \multirow[t]{3}{*}{ Rhoicosphenia sp. } & Planothidium sp. \\
\hline & Nedium sp. & & Cocconeis sp. \\
\hline & Cyclotella sp. & & \\
\hline \multirow[t]{5}{*}{ Green Algae } & $\begin{array}{ll}\text { Gongrosira } & \text { or } \\
\text { Apatococcus sp. } & \end{array}$ & \multirow[t]{5}{*}{ Spirogyra } & \multirow[t]{5}{*}{ Stigeoclonium } \\
\hline & Scenedesmus sp. & & \\
\hline & Monoraphidium sp. & & \\
\hline & Ankistrodesmus sp. & & \\
\hline & Unidentified colonial sp. & & \\
\hline \multirow[t]{4}{*}{ Blue-green Algae } & Oscillatoria sp. & \multirow[t]{4}{*}{ Oscillatoria sp. } & Oscillatoria sp. \\
\hline & Psuedanabaena sp. & & \multirow[t]{3}{*}{ Pseudanabaena sp. } \\
\hline & Leptolyngbya sp. & & \\
\hline & Phormidium sp. & & \\
\hline
\end{tabular}




\begin{tabular}{|c|c|c|c|}
\hline & Merismopedia $s p$. & & \\
\hline & $\begin{array}{ll}\text { Small } & \text { unidentified } \\
\text { colonial sp. } & \end{array}$ & & \\
\hline Desmids & Staurastrum sp. & Cosmarium sp. & Cosmarium sp. \\
\hline & & Closterium sp. & Closterium sp. \\
\hline & & & Staurastrum $s p$ \\
\hline
\end{tabular}

${ }^{1}$ diatoms listed generally from most abundant to least abundant

Note: Species composition included in this table is for reference only. Taxonomy was not conducted on periphyton plates used in the experiments presented here, however these compositions are fairly stable by season when periphyton plates of are similar gestation period.

6 Supplemental information on methods for ancillary aqueous uptake experiments with $N$.

7 triangulifer:

8 For aqueous uptake and efflux experiments, $N$. triangulifer larvae were transferred to individual 9 acid-washed exposure cups $(\mathrm{n}=5)$ with a small square PTFE substrate, filled with $25 \mathrm{~mL} \mathrm{pH}-$ 10 adjusted (7.2 \pm 0.2$) \mathrm{ASW}$ at nominal concentration of $10 \mu \mathrm{g} \mathrm{L} \mathrm{L}^{-1}$ arsenate along with ${ }^{73} \mathrm{As}$ as a 11 radiotracer $\left(0.005 \mu \mathrm{Ci} \mathrm{mL} \mathrm{mL}^{-1}\right)$. To obtain initial uptake rates from solutions, animals were 12 analyzed in vivo for radioactivity at 24,48 , and 72 hours following a rinse with concentration13 matched stable As (no radioisotope) solution to remove any superficially adsorbed radiotracer. 14 After the final time point, animals were returned to their exposure solutions for an additional 4-5 15 days of loading before being transferred to clean water to measure efflux. Individuals lost all 16 measureable radioactivity within the first 24 hours and therefore efflux could not be quantified. 